\title{
Increasing the efficiency of Organic Rankine Cycle Technology by means of Multivariable Predictive Control ${ }^{\star}$
}

\author{
Andres Hernandez*, Adriano Desideri**, Clara Ionescu*, \\ Sylvain Quoilin ${ }^{* *}$ Vincent Lemort and ${ }^{* *}$ Robin De Keyser* \\ * Department of Electrical energy, Systems and Automation, Ghent \\ University, Belgium (e-mail: Andres.Hernandez@UGent.be). \\ ** Thermodynamics Laboratory, University of Liege, Belgium.
}

\begin{abstract}
The Organic Rankine Cycle (ORC) technology has become very popular, as it is extremely suitable for waste heat recovery from low-grade heat sources. As the ORC system is a strongly coupled nonlinear multiple-input multiple-output (MIMO) process, conventional control strategies (e.g. PID) may not achieve satisfactory results. In this contribution our focus is on the accurate regulation of the superheating, in order to increase the efficiency of the cycle and to avoid the formation of liquid droplets that could damage the expander. To this end, a multivariable Model Predictive Control (MPC) strategy is proposed, its performance is compared to the one of PI controllers for the case of variable waste-heat source profiles.
\end{abstract}

Keywords: Model Predictive Control, Organic Rankine Cycle, Renewable energy.

\section{INTRODUCTION}

In recent years several studies have underlined the potential of low-grade heat recovery to reduce the amount of world-wide industrial energy consumption (IEA [2010]). The highly fluctuating nature of the heat source (temperature and mass flow) makes Waste Heat Recovery (WHR) applications a challenging task. Among the proposed solutions, Organic Rankine Cycle (ORC) systems stand out for their reliability and cost-effectiveness (Verneau [1979], Angelino et al. [1984]). The working principle is the same as a traditional steam Rankine cycle with the difference that water is replaced with an organic compound.

The studies on waste heat recovery ORC systems have been mainly focused on working fluid selection (Desai and Bandyopadhyay. [2009]), and system optimization (Sun and Li. [2011]). In the waste heat recovery context the ORC system undergoes fast transitions from nominal operating point to different off-design conditions, thus the development of a good control strategy in order to ensure optimal operation represents a challenging problem. Dynamic modeling is an important tool, necessary to analyze system dynamics and to test control strategies during transient and/or on-off conditions. Dynamic modeling of power plant has been studied in the last years, for energy conversion units (Colonna and Van Putten. [2007]), as well as for ORC systems (Casella et al. [2013]).

As far as safe operation is concerned, an accurate regulation of the superheating represents an important task for

\footnotetext{
* The results presented in this paper have been obtained within the frame of the IWT SBO-110006 project The Next Generation Organic Rankine Cycles (www.orcnext.be), funded by the Institute for the Promotion and Innovation by Science and Technology in Flanders. This financial support is gratefully acknowledged.
}

the controller. The regulator has to guarantee a minimum value of superheating in order to maximize the efficiency, and avoid the formation of liquid droplets at expander inlet that can damage the expansion machine (Wei et al. [2007]). In order to maximize the output power the evaporating pressure represents the most relevant controlled variable (Quoilin et al. [2011]). The main control objectives on control of Organic Rankine Cycles are then twofold: 1) Keep the cycle in a safe condition during operation and 2) maximize the net output power.

In (Quoilin et al. [2011]) three different decentralized control strategies based on PI controllers for varying heat source profiles are proposed, underlying that the best results are obtained with an evaporating temperature regulation based on a steady-state optimization. As the ORC power unit is a strongly coupled nonlinear multipleinput multiple-output (MIMO) system, conventional decentralized control strategies (e.g. PID) may not achieve satisfactory results. For this reason up to date work is related to the use of advance multivariable controllers. A Linear Quadratic Regulator (LQR) was implemented in (Zhang et al. [2012]), the controller exhibits a good tracking response to setpoint changes.

More recent developments include the use of advanced control strategies such as Model Predictive Control to improve the control of the ORC power unit. MPC is a general designation for controllers that make explicit use of a model of the plant to obtain an optimal control sequence by minimizing an objective function over a defined prediction horizon. In MPC only the first value of the calculated control signal is applied to the system, then the entire procedure is repeated the next sampling time i.e. 'receding horizon principle'. In (Hou et al. [2011]) a supervisory predictive control is applied, in (Changenet 
et al. [2008], Lemort et al. [2011]) a Predictive Functional Control (PFC) is tested for controlling the evaporator superheat. In (Zhang et al. [2013]) the ability of a generalized predictive control (GPC) to track a reference and to reject disturbances is tested. Both studies are restricted to evaluate the tracking capabilities of the different multivariable controllers only in the case of step disturbances and no attention is paid to the performance of the power unit.

In this study the Extended Prediction Self-Adaptive Control (EPSAC) approach to MPC for MIMO systems (De Keyser [2003]) is implemented to increase the cycle efficiency by accurately regulating the superheating and evaporating temperature, of an ORC unit for WHR application. The proposed strategy is compared to a more traditional approach based on decentralized PI control.

The paper is structured as follows: in section 2 the architecture and main characteristics of the ORC system used in this study is presented. Next, in section 3 the EPSAC methodology is briefly described. The control design of the multivariable MPC as well as the decentralized PI strategy is described in section 4 , followed by the simulation results in section 5. Finally a conclusion section summarizes the main outcome of this contribution.

\section{SYSTEM DESCRIPTION}

This section describes the architecture and the main characteristics of the ORC system model used for evaluating the performance of the developed control strategy.

\subsection{The Organic Rankine Cycle System}

A schematic layout of the ORC system is presented in Fig. 1.

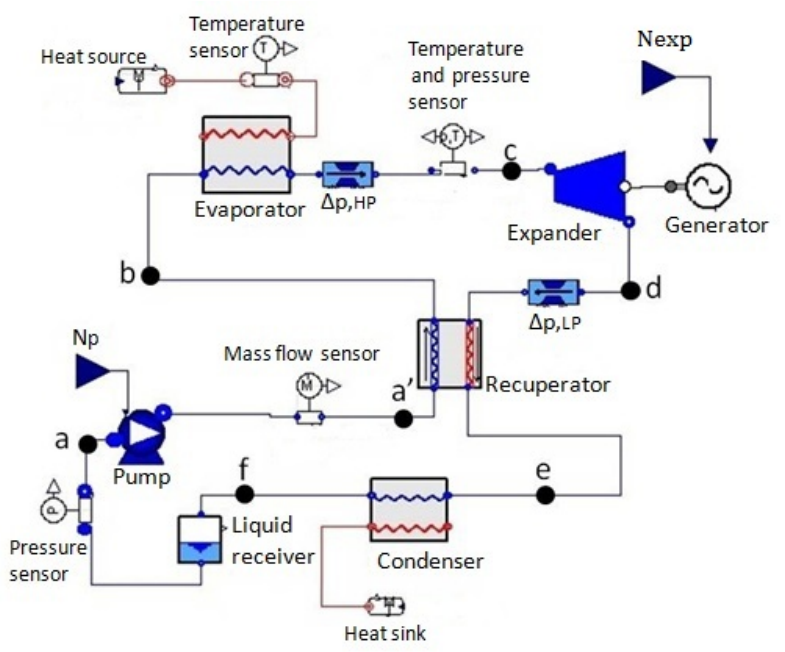

Fig. 1. Schematic layout of the setup from the Dymola GUI

The system model considered in this study is based on the pilot plant size available at the Howest college in Kortrijk (Belgium). The system has a nominal power of $6.5 \mathrm{kWe}$ and it is based on a regenerative cycle. The expander is originally a single screw compressor adapted to run in expander mode. For simulation purposes the refrigerant
R245fa is selected as working fluid whilst the pilot plant works with solkatherm.

Referring to the left side of figure 1, it is possible to recognize the liquid receiver installed at the outlet of the condenser (a), where the fluid is collected in saturated liquid condition (f). From the receiver outlet (a), the fluid is pumped through the regenerator cold side (a'), and the evaporator (b), where it is heated up to superheated vapor, reaching its maximum temperature $\mathrm{T}_{c}$ at the evaporator outlet (c). The fluid, after being expanded in the volumetric machine, enters the regenerator hot side (d), and then it flows into the condenser (e).

As discussed above a minimum amount of superheating at expander inlet is required to mantain safe operation. The superheating is defined as:

$$
\Delta T_{e x p, e v}=T_{e x p, s u}-T_{e v}
$$

where $T_{e x p, s u}$ is the temperature measured at the inlet of the expander and $T_{e v}$, the evaporating temperature, corresponding to the temperature at which the fluid undergoes the phase transition from saturated liquid to saturated vapor at the fixed evaporating pressure $P_{e v}$.

$$
T_{e v}=f\left(P_{e v}\right)
$$

where $f$ corresponds to a function that correlates the pressure for the refrigerant R245FA (Quoilin et al. [2011]).

The main terms to assess the performance of the ORC system are the net output power and the cycle efficiency which are defined in equation (3) and (4) respectively.

$$
\begin{gathered}
\dot{W}_{e l, n e t}=\dot{W}_{\text {exp }}-\dot{W}_{\text {pump }} \\
\eta_{\text {cycle }}=\frac{\dot{W}_{\text {el,net }}}{\dot{Q}_{\text {in }, \text { ORC }}}
\end{gathered}
$$

where $\dot{W}_{\text {exp }}$ is the expander electrical power, $\dot{W}_{\text {pump }}$ is the pump electrical power and $\dot{Q}_{i n, O R C}$ is the thermal power supplied to the ORC system in the evaporator.

The optimal working conditions for an ORC system are listed hereunder:

- The condensing pressure should be maintained as low as possible.

- The superheating at evaporator outlet (c) has to be maintained as low as possible.

- An optimization of the overall efficiency result in the optimal evaporation temperature.

Two degrees of freedom are available to match these optimal working conditions: the pump speed and the expander speed (Quoilin et al. [2011]). This study focuses on the application of two different control strategies to accurately regulate the evaporating temperature and superheating by acting on both degrees of freedom.

In order to assess the performance of the different developed control strategies a dynamic model of the ORC system (Fig. 1) has been developed in the Modelica language using existent components from the ThermoCycle library (Quoilin et al. [2014]). The developed Modelica model is

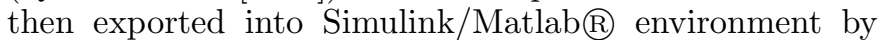
means of the Functional Mock-Up Interface (FMI) open standard. In this study the FMI Toolbox provided by Modelon( $\mathrm{R}$ was used to compile the dymola model in a model exchange format. 


\subsection{Low-order Model suitable for Prediction}

The model required by MPC has been obtained based on a parametric identification procedure. Four transfer functions representing the system dynamics between the inputs, pump $\left(N_{p}\right)$ and expander $\left(N_{\text {exp }}\right)$ speed, and the outputs, superheating $\left(\Delta T_{e x p, e v}\right)$ and evaporating temperature $\left(T_{e v}\right)$, were obtained around the nominal operating conditions presented in table 1 . In the pilot plant setup, the speed in the pump $\left(N_{p}\right)$ and expander $\left(N_{e} x p\right)$ are controlled by means of inverters, so that the user provide the speed in revolutions per minute (rpm).

Table 1. Nominal operating conditions considered for the Identification Procedure

\begin{tabular}{cccc}
\hline Parameter & Description & Value & Unit \\
\hline$N_{p}$ & Pump rotational speed & 1710 & $\mathrm{rpm}$ \\
$N_{\text {exp }}$ & Expander rotational speed & 2880 & $\mathrm{rpm}$ \\
$T_{\text {ev }}$ & Evaporating temperature & 401 & $\mathrm{~K}$ \\
$\Delta T_{\text {exp,ev }}$ & Superheating & 15 & $\mathrm{~K}$ \\
$T_{h f}$ & Temperature hot fluid & 145 & ${ }^{\circ} C$ \\
$\dot{M}_{h f}$ & Mass flow rate hot fluid & 3 & $\mathrm{~kg} / \mathrm{s}$ \\
$T_{c f}$ & Temperature cold fluid & 20 & ${ }^{\circ} C$ \\
$\dot{M}_{c f}$ & Mass flow rate cold fluid & 4 & $\mathrm{~kg} / \mathrm{s}$ \\
$\dot{W}_{\text {el }, \text { net }}$ & Net output power & 6.5 & $\mathrm{~kW}$ \\
$\eta_{c y c l e}$ & Cycle efficiency & 6 & $\%$ \\
\hline
\end{tabular}

The identification has been performed using a multisine excitation signal and the prediction error method (pem) (Ljung [2007]). The sampling time $T_{s}=5 \mathrm{~s}$ has been chosen according to the fastest dynamics of the system. The identified transfer function matrix in deviation variables is presented in (5).

$$
\left[\begin{array}{c}
\overline{\Delta T_{\text {exp }, e v}}(s) \\
\overline{T_{e v}}(s)
\end{array}\right]=\left[\begin{array}{cc}
\frac{-4.5036}{(141.2 s+1)} & \frac{1.0351}{(104.56 s+1)} \\
\frac{1.37}{(134.2 s+1)} & \frac{-0.48}{(54.14 s+1)}
\end{array}\right]\left[\begin{array}{c}
\overline{N_{p}}(s) \\
\overline{N_{e x p}}(s)
\end{array}\right]
$$

The normalized root-mean-square error of each transfer function to validation data was computed using (6):

$$
f i t=100 *\left(1-\frac{\|y-\hat{y}\|}{\|y-\operatorname{mean}(y)\|}\right)
$$

where $y$ is the validation data output and $\hat{y}$ is the model output given by the transfer function. Each transfer function gave a data fitting of about $80 \%$

\section{MULTIVARIABLE EPSAC METHOD}

This section briefly summarizes the extension of the Extended Prediction Self-Adaptive Control (EPSAC) for multivariable systems, following the 'solidary' approach (De Keyser [2003]).

\subsection{Principle of MIMO EPSAC}

The analysis of the method is described considering the case of a $n_{u}$ inputs and $n_{y}$ outputs. The following structure of the generic process model is used:

$$
y_{i}(t)=x_{i}(t)+n_{i}(t), i=1,2, \ldots, n_{y}
$$

with $y_{i}(t)$ the measured process outputs, $x_{i}(t)$ the model outputs and $n_{i}(t)$ the process/model disturbances (i.e. modeling errors and noise); $t$ denoting the discrete-time

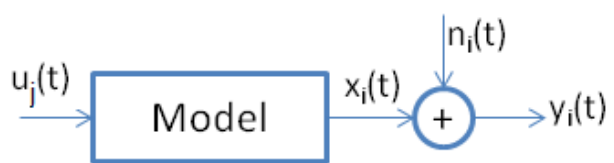

Fig. 2. Process Model

index. A schematic representation is depicted in Fig. 2 where $u_{j}(t)$ represent the process inputs $j=1, \ldots, n_{u}$.

The disturbance $n_{i}(t)$ includes all effects in the measured output $y_{i}(t)$ which do not come from the model output $x_{i}(t)$. This is a fictitious (and thus non-measurable) signal, which can be modeled by a colored noise process:

$$
n_{i}(t)=\frac{C\left(q^{-1}\right)}{D\left(q^{-1}\right)} e(t)
$$

with $e(t)$ white noise; $C\left(q^{-1}\right)$ and $D\left(q^{-1}\right)$ (monic polynomials in the shift operator $q$ ).

An essential aspect in the MPC methodology consists of the prediction of the process outputs, these are calculated with:

$$
y_{i}(t+k \mid t)=x_{i}(t+k \mid t)+n_{i}(t+k \mid t), i=1,2, \ldots, n_{y}
$$

for $k=N_{1 i}, \ldots, N_{2 i}$ where $N_{1 i}$ and $N_{2 i}$ are the minimum and the maximum prediction horizons for each $i$-output of the process. Our problem resides now on finding $x_{i}(t+k \mid t)$ and $n_{i}(t+k \mid t)$. The first multi-step prediction problem is solved by recursion of the process model, while the second is solved using filtering techniques on the disturbance model. A detailed description is given in (De Keyser [2003]).

The future response of the process is considered to be the result of two effects:

$$
y_{i}(t+k \mid t)=y_{\text {ibase }}(t+k \mid t)+y_{\text {iopt }}(t+k \mid t), i=1,2, \ldots, n_{y}
$$

The two contributions have the following origins:

$y_{\text {ibase }}(t+k \mid t)$ :

- effect of past controls and of the basic future control scenario, called $u_{\text {jbase }}(t+k \mid t)$, for $k=0 \ldots, N_{u j}-1$ ( $N_{u}$ being the control horizon), and for $j=1 \ldots n_{u}$. For linear systems the choice of this scenario is irrelevant, a simple choice being $u_{j b a s e}(t+k \mid t) \equiv 0, k \geq 0$

- effect of future (predicted) disturbances $n_{i}(t+k \mid t)$.

$\underline{y_{\text {iopt }}(t+k \mid t)}$ :

- effect of the optimizing future control actions: $\delta u_{j}(t+$ $k \mid t)=u_{j}(t+k \mid t)-u_{j b a s e}(t+k \mid t), k=0 \ldots N_{u}-1$. Where $u_{j}(t+k \mid t)$ are the desired optimal control actions. The effect of these additions is the discrete time convolution of $\Delta U_{j}=\left\{\delta u_{j}(t \mid t), \ldots, \delta u_{j}\left(t+N_{u}-\right.\right.$ $1 \mid t)\}$ with the impulse response coefficients of the system ( $\mathrm{G}$ matrix).

In brief the key EPSAC-MPC equations for the MIMO case (10), can be expressed in matrix notation:

$$
\mathbf{Y}_{\mathbf{i}}=\mathbf{Y}_{\mathbf{i B a s e}}+\mathbf{Y}_{\mathbf{i o p t}}=\overline{\mathbf{Y}}_{\mathbf{i}}+\sum_{\mathbf{j}=\mathbf{1}}^{\mathbf{n}_{\mathbf{u}}} \mathbf{G}_{\mathbf{i j}} \mathbf{U}_{\mathbf{j}}
$$

where for $i=1,2, \ldots, n_{y}$ and $j=1,2, \ldots, n_{u}$ : 


$$
\begin{aligned}
\mathbf{Y}_{\mathbf{i}} & =\left[y_{i}\left(t+N_{1 i} \mid t\right) \ldots y_{i}\left(t+N_{2 i} \mid t\right)\right]^{T} \\
\overline{\mathbf{Y}}_{\mathbf{i}} & =\left[y_{\text {ibase }}\left(t+N_{1 i} \mid t\right) \ldots y_{\text {ibase }}\left(t+N_{2 i} \mid t\right)\right]^{T} \\
\mathbf{U}_{\mathbf{j}} & =\left[\delta u_{j}(t \mid t) \ldots \delta u_{j}\left(t+N_{u j}-1 \mid t\right)\right]^{T} \\
\mathbf{G}_{\mathbf{i j}} & =\left[\begin{array}{cccc}
h_{N_{1 i}}^{i j} & h_{N_{1 i}-1}^{i j} & \ldots & g_{N_{1 i}-N_{u j}+1}^{i j} \\
h_{N_{1 i}+1}^{i j} & h_{N_{1 i}}^{i j} & \ldots & \ldots \\
\ldots & \ldots & \ldots & \ldots \\
h_{N_{2 i}}^{i j} & h_{N_{2 i}-1}^{i j} & \ldots & g_{N_{2 i}-N_{u j}+1}^{i j}
\end{array}\right]
\end{aligned}
$$

\subsection{The Control Objective}

Without affecting the generality of the problem a system with 2 inputs and 2 outputs will be considered in the next analysis. The objective is to find the optimal control vectors $\Delta U_{1}^{*}$ and $\Delta U_{2}^{*}$ which minimize the cost function:

$J=\sum_{k=N_{11}}^{N_{21}}\left[r_{1}(t+k \mid t)-y_{1}(t+k \mid t)\right]^{2}+\sum_{k=N_{12}}^{N_{22}}\left[r_{2}(t+k \mid t)-y_{2}(t+k \mid t)\right]^{2}$

subject to

$$
\begin{aligned}
\Delta u_{j \min } & \leq \Delta u_{j}(t+k \mid t) \leq \Delta u_{j \max } \forall k=0, \ldots, N_{u j}-1 \\
u_{\text {jmin }} & \leq u_{j}(t+k \mid t) \leq u_{\text {jmax }} \forall k=0, \ldots, N_{u j}-1
\end{aligned}
$$

where $N_{2 i}$ is the prediction horizon; $N_{u j}$ is the control horizon; $r_{i}(t+k \mid t)$ is the future reference sequence; $u(t+$ $k \mid t)$ the optimal future control sequence; $\Delta u_{j}(t+k \mid t)$ is the incremental control action; $y_{i}(t+k \mid t)$ is the $k$ step ahead prediction of the system output $y(t)$ on data up to time $t$ for outputs $i=1,2$ and inputs $j=1,2$.

The desired reference trajectory $r_{i}(t+k \mid t)$, is chosen here as a $1^{\text {st }}$-order reference trajectory as specific implementation example:

$$
r_{i}(t+k \mid t)=\alpha r_{i}(t+k-1 \mid t)+(1-\alpha) w_{i}(t+k \mid t)
$$

for $k=1 \ldots N_{2}$ and initialization $r(t \mid t)=y(t)$. The signal $w(t)$ represents the setpoint and $\alpha$ a design parameter that plays an important role in tuning the MPC performance (Sanchez and Rodellar. [1996]).

With this strategy the predicted control errors summed over all process outputs are minimized. Notice that the control error for a specific variable $y_{1}$ can possibly and deliberately be increased, with the purpose of reducing the control error for another variable $y_{2}$. The objective is thus to minimize the total control error of all partners together, and not just to minimize the individual control error of each partner separately; hence, the choice for the name solidary control.

The cost function (12) can be represented as a quadratic cost index in $\Delta U$, for which constraints can be taken into account a priori, thus leading to the best solution that is possible within the specified limits. Above problem is a standard, well-known optimization problem called quadratic programming (quadratic cost function with linear inequality constraints) (De Keyser [2003]).

\section{CONTROLLER STRUCTURE AND TUNING}

In this section the control structure and tuning procedure of both the decentralized PI control strategy and the proposed multivariable MPC is discussed.

\subsection{Multivariable EPSAC design}

The input $u_{j}(t)$ and slew rate $\Delta u_{j}(t)$ constraints imposed to the developed MPC are presented in table 2 .

Table 2. Input Constraints of ORC system

\begin{tabular}{ccccc}
\hline Input & Initial Value & $u_{\max }$ & $u_{\min }$ & $\Delta u_{\max }$ \\
\hline$N_{p}$ & $1710 \mathrm{rpm}$ & $2910 \mathrm{rpm}$ & $510 \mathrm{rpm}$ & $120 \mathrm{rpm} / \mathrm{s}$ \\
$N_{\text {exp }}$ & $2880 \mathrm{rpm}$ & $3840 \mathrm{rpm}$ & $1920 \mathrm{rpm}$ & $120 \mathrm{rpm} / \mathrm{s}$ \\
\hline
\end{tabular}

In MPC, a balance between acceptable control effort and acceptable control error can be obtained via many design parameters (e.g. the reference trajectory design parameter $\alpha$; the prediction horizon $N_{2}$ and the control horizon design parameter $N_{u}$ ). Fast or slow responses can be obtained by a low or high $N_{2}$, respectively. The control horizon $N_{u}$ is used to structure the future control scenario, reducing the degrees of freedom from $N_{2}$ to $N_{u}$. Structuring leads to simplified calculations and has generally a positive effect on robustness. The design parameter $\alpha$ in the reference trajectory can vary in the range of: $0 \leq \alpha \leq 1$. A value of $\alpha$ closer to 1 means a smoother variation of the setpoint and hence a less aggressive control action.

Several simulations have been performed varying the design parameters $N_{2}, N_{u}$ and $\alpha$. A trade-off between closed loop speed and robustness has been obtained for $\{60,1,0.5\}$, respectively. The main goal is to achieve a response without overshoot $O S \%=0$ and settling time of about $300 \mathrm{~s}$. Another important element in the design of the controller is the choice of the disturbance model (8), during this study the 'default' filter $\frac{C\left(q^{-1}\right)}{D\left(q^{-1}\right)}=\frac{1}{1-q^{-1}}$ has been chosen leading to zero steady-state error.

The complete control scheme is depicted in Fig. 3, where the blue blocks represent the proposed MPC strategy.

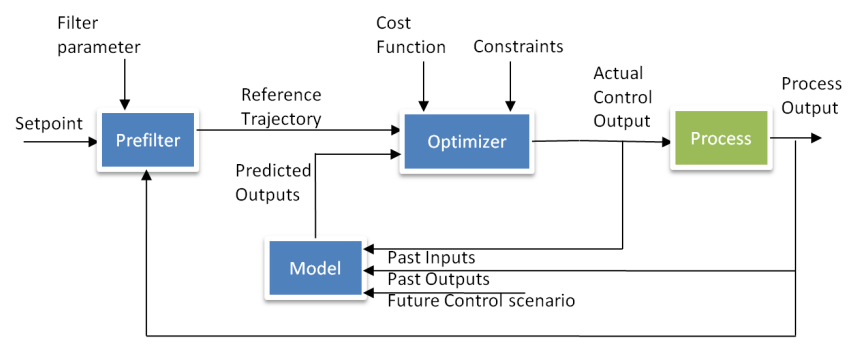

Fig. 3. Multivariable EPSAC control structure

\subsection{Decentralized PI control design}

Two PI controllers following a decentralized scheme (Fig. 4) have been implemented as a reference to the performance of the MPC. This approach has been chosen since it represents the typical control strategy adopted for ORC systems (Quoilin et al. [2011]).

Using the model (5) the two PI controllers were tuned using the CAD tool FRTool in matlab (De Keyser and Ionescu. [2006]), for the following specifications: settling time of $300 \mathrm{~s}$ overshoot percent $O S \%=0$ and robustness $R o=0.7$. The PI parameters obtained for superheating $\Delta T_{\text {exp }, \text { ev }}$ are $K p=-0.4055, T i=73.6068$ and the PI 


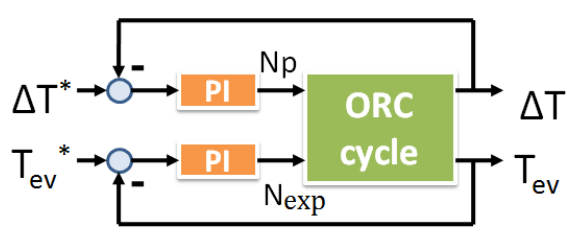

Fig. 4. Decentralized PI control structure

parameters to control the evaporating temperature $T_{e v}$ are $K p=-0.9807, T i=17.4471$.

\subsection{Setpoint tracking experiment}

As above mentioned the present study focuses on the accurate regulation of the superheating $\Delta T_{e x p, e v}$, where a small amount of superheating represents a higher efficiency (Wei et al. [2007]) and zero represents an undesired effect (i.e. formation of liquid droplets that could damage the expander).

In order to compare the MPC strategy with the more traditional PI, a tracking experiment is proposed as depicted in Fig. 4.3. At time $1500 \mathrm{~s}$ the setpoint of superheating is changed from $15 \mathrm{~K}$ to $13 \mathrm{~K}$, the MPC controller exhibits a better response with less oscillations and less interaction in the other output (i.e. better decoupling effect than the PI controller). Next, a change in the setpoint of the evaporating temperature is made, from $401.2 \mathrm{~K}$ to $400.5 \mathrm{~K}$, both controllers present a settling time of about $400 \mathrm{~s}$ but the PI strategy requires a minor control effort compared to the MPC one.
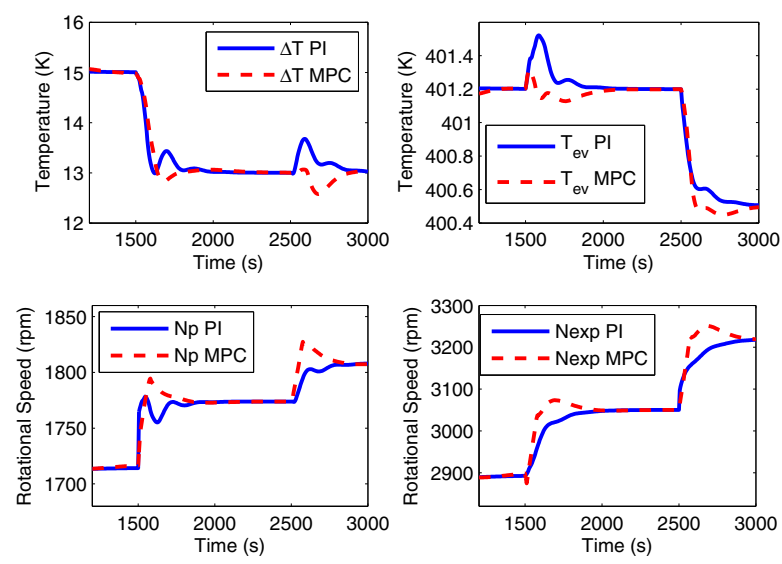

Fig. 5. Tracking performance of PIs 'continuous blue line' and MIMO MPC 'dashed red line'

From this experiment some preliminary conclusions can be drawn. As expected, the MPC strategy allows for a good decoupling of the system since it is a multivariable strategy, which 'knows' by means of the model the expected interactions in the system. As far as robustness is considered, both linear strategies lead to similar performance after the change in the setpoint. The robustness of the control strategies was considered by tuning a slow decentralized PI controller and by selecting a large prediction horizon $N_{2}$ (to deal with modeling errors during prediction). Finally, the MPC strategy presents a desired response while respecting the input constraints imposed by the actuators.

\section{SIMULATION RESULTS}

The experiment presented in this section consists in analyzing which strategy is the best in terms of keeping a high cycle efficiency and delivering the maximum net output electrical power, in case of unsteady condition of the thermal energy source (i.e. temperature and mass flow rate variations).
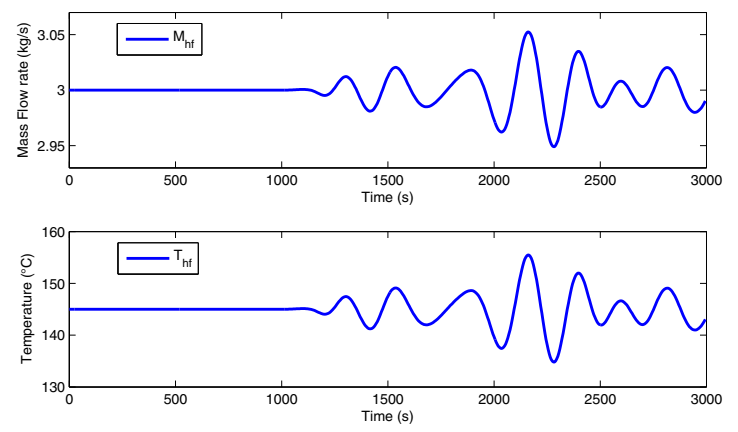

Fig. 6. Temperature and mass flow rate variations of the heat source

The mass flow rate and temperature of the heat source $M_{h f}$ and $T_{h f}$, respectively, are considered to be constant during the first $1200 \mathrm{~s}$; afterwards, small variations are introduced from $1200 \mathrm{~s}$ to $2000 \mathrm{~s}$, then larger variations are introduced from $2000 \mathrm{~s}$ to $2500 \mathrm{~s}$. Finally, the variations are again diminished until the end of the experiment. The obtained heat source profile is depicted in Fig. 6 .
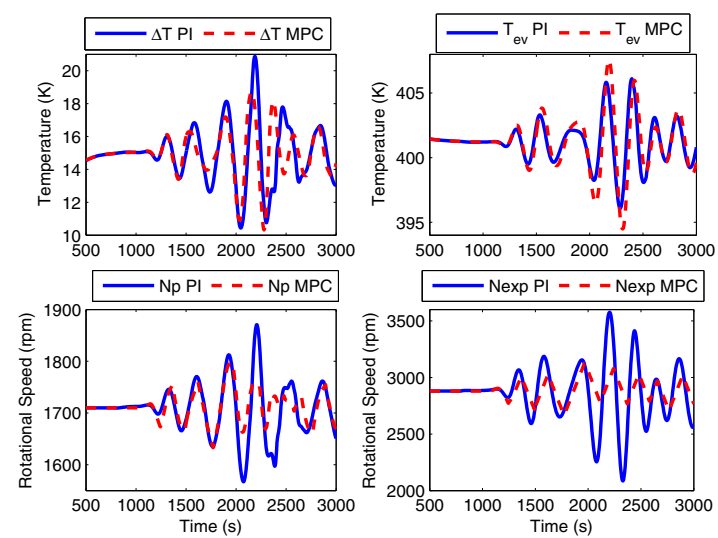

Fig. 7. Disturbance rejection performance of PIs 'continuous blue line' and MIMO MPC 'dashed red line'

The results for both strategies are depicted in Fig. 7. An important conclusion is that for a constant thermal energy source profile both PI and MPC strategies are equivalent in terms of performance as observed during the first $1200 \mathrm{~s}$. However, for a large variation in the variable heat source profile (e.g. between $2000 \mathrm{~s}$ and $2500 \mathrm{~s}$ ) the MPC strategy shows a better perfomance than the PI controllers keeping the superheating $\Delta T_{\text {exp ev }}$ closer to the setpoint, with considerably less control effort (Fig. 7). As a consequence the produced net electrical power and hence the thermal cycle efficiency of the system, based on the MPC strategy, results affected by smaller oscillations compared to the PI controlled system as shown in Fig. 8.

Similar to the previous analysis, in Fig. 8 both strategies deliver the same electrical power for small variations in 
the heat source profile; nevertheless, for large variations the MPC strategy leads to a higher average efficiency and consequently a higher average net electrical output power with less control effort.
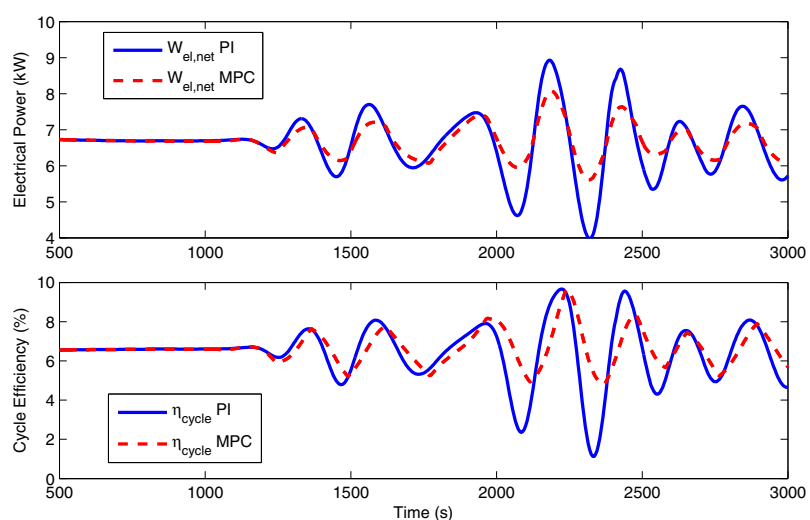

Fig. 8. Electrical power produced and cycle efficiency. For both decentralized PIs 'blue continuous line' and MIMO MPC 'dashed red line.'

\section{CONCLUSION}

In the present contribution the effectiveness of the proposed Multivariable Predictive Control strategy has been evaluated and compared to PI controllers. The results obtained suggest that: 1) In case of stable thermal energy source, classical control techniques (e.g. PID) gives similar performance compared to more advanced strategies (e.g. MPC). 2) In case of unsteady thermal energy source, a characteristic of waste heat recovery applications, the MPC allows to reach a higher net electrical output power, by more accurately regulating the superheating, leading to higher efficiency of the cycle compared to a traditional PI strategy.

Future work include adding a degree of freedom by controlling the condenser pressure by acting on the mass flow rate and/or temperature of the heat sink, $M_{c f}$ and $T_{c f}$ respectively. Development of more advanced control to maximize the efficiency in off-design working conditions, by optimizing the evaporating temperature are also under investigation.

\section{REFERENCES}

G. Angelino, M. Gaia, and E. Macchi. A review of italian activity in the field of organic rankine cycles. In proceedings of the International VDI-Seminar, pages 465-482, 1984.

F. Casella, T. Mathijssen, P. Colonna, and J. Van Buijtenen. Dynamic modeling of organic rankine cycle power systems. Journal of Engineering for Gas Turbines and Power, Vol 135:042310-042310-12, 2013.

C. Changenet, J.N. Carvet, D. Gehin, F. Sicard, and B. Charmel. Study on predictive functional control of an expansion valve for controlling the evaporator superheat. J. Systems and Control Engineering, Vol. 222 Part I.:571-582, 2008.

P. Colonna and H. Van Putten. Dynamic modeling of steam power cycles.: Part i modeling paradigm and validation. Applied Thermal Engineering, 27(2-3):467 $-480,2007$. ISSN 1359-4311.
R. De Keyser. Model based Predictive Control for Linear Systems, chapter invited in UNESCO Encyclopaedia of Life Support Systems (EoLSS). UNESCO Encyclopaedia of Life Support Systems http://www.eolss.net Article contribution 6.43.16.1 (available online at: http://www.eolss.net/samplechapters/c18/e6-43-16-01.pdf) Eolss Publishers Co Ltd, Oxford, 35 pages, 2003.

R. De Keyser and C.M Ionescu. A frequency response tool for cacsd in matlab. In IEEE International Symposium on Computer Aided Control Systems Design, pages 2275-2280, Munich, 2006.

N. Desai and S. Bandyopadhyay. Process integration of organic rankine cycle. J. Energy, Vol. 34:1674-1686, 2009.

G. Hou, R. Sun, G. Hu, and J. Zhang. Supervisory predictive control for evaporator in organic rankine cycle (orc) system for waste heat recovery. In Proceedings of the 2011 International Conference on Advanced Mechatronics Systems, pages 306-311, 2011.

IEA. Industrial excess heat recovery technologies \& applications. Technical report, Technical report, Industrial Energy-related Technologies and Systems (IETS), 2010.

V. Lemort, A. Zoughaib, and S. Quoilin. Comparison of control strategies for waste heat recovery organic rankine cycle systems. In Sustainable Thermal Energy Management in the Process Industries International Conference (SusTEM2011), 2011.

L. Ljung. System identification: theory for the user. Prentice-Hall, 2007.

S. Quoilin, R. Aumann, A. Grill, A. Schuster, and V. Lemort. Dynamic modeling and optimal control strategy for waste heat recovery organic rankine cycles. Applied Energy, Vol. 88:2183-2190, 2011.

S. Quoilin, A. Desideri, J. Wronski, I. Bell, and V. Lemort. Thermocycle: A modelica library for the simulation of thermodynamic systems. In Proceedings of the 10th International Modelica Conference, Lund, Sweden, 2014.

M. Sanchez and J. Rodellar. Adaptive Predictive Control. ISBN 0135148618. Prentice Hall London, 1996.

J. Sun and W. Li. Operation optimization of an organic rankine cycle (orc) heat recovery power plant. J. Applied Thermal Engineering, Vol. 31:2032-2041, 2011.

A. Verneau. Waste heat recovery by organic fluid rankine cycle. In In Proceedings from the First Industrial Energy Technology Conference, pages 940-952, Houston, TX, 1979.

D. Wei, X. Lu, Z. Lu, and J. Gu. Performance analysis and optimization of organic rankine cycle (orc) for waste heat recovery. J. Energy Conversion and Management, Vol. 48:1113-1119, 2007.

J. Zhang, Y. Zhou, S. Gao, and G. Hou. Dynamic modeling and multivariable control of organi rankine ccycle in waste heat utilizing processes. Computers and Mathematics with Applications, 64:908-921, 2012.

J. Zhang, Y. Zhou, Y. Li, G. Hou, and F. Fang. Generalized predictive control applied in waste heat recovery power plants. Applied energy, 102:320-326, 2013. 